\title{
Early diagnosis of breast angiosarcoma
}

Authors: Vana Stojić ${ }^{1}$, Tea Štrbac ${ }^{1}$, Petra Sulić ${ }^{2}$, Stjepan Frkanec ${ }^{1}$, Zvonko Zadro $^{3}$ (Mentor)

${ }^{1}$ University of Zagreb, School of Medicine, Zagreb, Croatia

${ }^{2}$ Petrovčić Marija Ordinacija Opće Medicine, Albaharijeva BB, 10000 Zagreb, Grad Zagreb

${ }^{3}$ Department of Plastic and Reconstructive Surgery, Clinical Hospital "Sveti Duh", Zagreb, Croatia

DOI: https://doi.org/10.26800/LV-142-supp5-6

\section{Background:}

Angiosarcoma is a rare and aggressive soft tissue breast cancer. It occurs in a primary form without a known precursor, typically in younger patients. In a secondary form, it is associated with a history of irradiated breast tissue, and it usually occurs in older patients. It can happen as many as 8-10 years after getting radiation treatment to the breast. When combined with chronic lymphedema, secondary angiosarcoma in an edematous limb after mastectomy and radiotherapy is referred to as StewartTreves syndrome. Secondary angiosarcomas spread to the parenchyma, while primary forms arise in the parenchyma.

\section{Case report:}

We present a 60-year-old woman with angiosarcoma grade III on the left breast. It presented as an exulcerated bleeding mass $10 \mathrm{~cm}$ in diameter with enlarged axillary lymph nodes. She noticed a lump in that area about a month before. The patient has no history of irradiation. Ultrasound showed a $\mathrm{BI}$ RADS 5 lesion. Thoracal MSCT revealed an expansive pathologic substrate in the upper outer quadrant of the left breast. It was not sure if it extends to axillary lymph nodes. The tumor was estrogen/progesterone-receptor negative. Tumor markers CEA, CA 15-3, CA 125 were at normal levels. An extempore biopsy was made in general anesthesia. After positive histopathologic results, the left breast was ablated. Lymph nodes were extirpated three months later. Further oncological investigation and additional treatment are required.

\section{Conclusion:}

Even though breast angiosarcoma is rare compared to carcinoma, we should not forget about it. Early diagnosis of angiosarcoma is essential for a favorable prognosis. As the use of breast conservative therapy has increased over the last 30 years, it is assumed that the incidence of secondary angiosarcoma will likely increase.

Keywords: Angiosarcoma, Breast, Cancer 